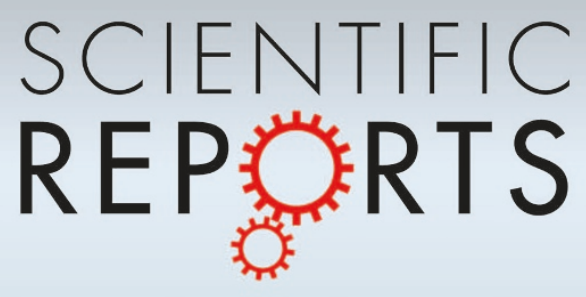

OPEN

SUBJECT AREAS:

NANOPHOTONICS AND

PLASMONICS

QUANTUM OPTICS

NANOWIRES

ATOMIC AND MOLECULAR INTERACTIONS WITH

PHOTONS

Received

12 March 2013

Accepted

3 September 2013

Published

24 September 2013

Correspondence and requests for materials should be addressed to

G.C.F. Igerard.colasdes-francs@ubourgogne.fr)

\section{Coupling of a dipolar emitter into one-dimensional surface plasmon}

\author{
Julien Barthes, Alexandre Bouhelier, Alain Dereux \& Gérard Colas des Francs \\ Laboratoire Interdisciplinaire Carnot de Bourgogne (ICB) UMR 6303 CNRS/Université de Bourgogne. 9, Av. Savary, BP 47870, \\ 21078 Dijon Cedex, FRANCE.
}

Quantum plasmonics relies on a new paradigm for light-matter interaction. It benefits from strong confinement of surface plasmon polaritons (SPP) that ensures efficient coupling at a deep subwavelength scale, instead of working with a long lifetime cavity polariton that increases the duration of interaction. The large bandwidth and the strong confinement of one dimensional SPP enable controlled manipulation of a nearby quantum emitter. This paves the way to ultrafast nanooptical devices. However, the large SPP bandwidth originates from strong losses so that a clear understanding of the coupling process is needed. In this report, we investigate in details the coupling between a single emitter and a plasmonic nanowire, but also SPP mediated coupling between two emitters. We notably clarify the role of losses in the Purcell factor, unavoidable to achieve nanoscale confinement down to $10^{-4}(\lambda / n)^{3}$. Both the retarded and band-edge quasi-static regimes are discussed.

ight matter interaction at the single atom/photon level is extremely weak and strategies have to be developed to enhance this coupling. In a simple picture, the coupling strength depends on the quality factor $Q$ and the - effective volume $V$ of the involved mode by the ratio $Q / V$. High $Q / V$ ratio, hence efficient light-matter interaction, is achieved in cavity-based systems, where large modal volumes are easily compensated by extremely narrow resonances ${ }^{1,2}$. It has been also proposed to work with diffraction limited volumes by coupling a singleatom to an elongated fiber ${ }^{3}$. Another downscaling step is made with plasmonics ${ }^{4}$. Surface plasmon polariton (SPP) results from coupling electromagnetic wave to a surface density of charges. They are therefore naturally confined near a metal surface and are not diffraction limited, offering thus a new tool for interfacing light and matter at the nanometer scale $e^{5-7}$. Since the seminal work of Chang et al. ${ }^{8}$, coupling between a dipolar emitter and a metal nanowire received strong interest. Metal nanowires define 1D plasmonic waveguides with a great potential for integrated optical routing'. They can be chemically synthesized with high crystallinity hence supporting SPP with reduced losses ${ }^{10-12}$. Controlled positionning of quantum emitter(s) near 1D plasmonic guide is now achievable $\mathrm{e}^{13-15}$, opening the way to realize original nano-optical devices. For instance, SPP propagation can be controlled by a single emitter, leading to the concept of single photon transistor ${ }^{16}$. Reciprocally, two distant emitters can be interfaced via surface plasmons, with applications such as SPP mediated resonant energy transfer ${ }^{17}$, remote qubits entanglement ${ }^{18,19}$ or nano-optical logical gates ${ }^{20}$.

This report is devoted to a careful analysis of the coupling mechanisms between one or two individual emitters and a metal nanowire. We quantify all the coupling channels (radiative and non radiative coupling, surface plasmon coupled emission, electron scattering). To date, radiative and non radiative rates were estimated within the quasistatic approximation ${ }^{8}$. Moreover, the expression of the decay rate into plasmon modes were derived in the lossless case only ${ }^{8,21}$. In this context, we are specifically interested in the role of losses in the emitter-SPP coupling mechanism. Therefore, we investigate two distinct regimes. A first regime taking into account retardation explaining SPP propagation and routing, and a second regime focused on quasi-static SPP responsible for an efficient interfacing with dipolar emitters but at the price of strong losses. To this aim, we consider a gold nanowire since both retardation regime and quasi-static pictures are present in the optical domain. Figure 1 represents the studied configuration. A dipolar emitter excites a surface plasmon supported by a gold nanowire. The report is organized as follows. We first carefully describe the modes properties of a 1D plasmonic nanowire in presence of losses. This allows for a rigorous description of the coupled emitter-nanowire system in the retarded regime. We identify all the relaxation channels of the emitter close to $1 \mathrm{D}$ plasmonic nanowire and compare our data to previous works. We notably achieve a closed form expression for surface plasmon coupled emission, in presence of losses with a clear link to the Purcell factor. This closed form expression permits to unambiguously define the mode volume of lossy 1D SPP. Then, we discuss SPP mediated dipole-dipole coupling between two 


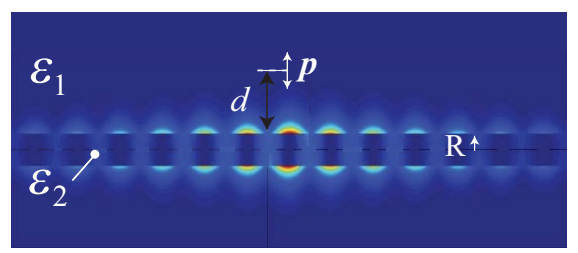

Figure 1 Surface plasmon coupled emission of a dipolar quantum emitter close to a metal nanowire of radius $\mathrm{R}$. We represent the intensity at a given instant of the guided SPP. $\mathbf{p}$ refers to the emitter dipole moment and $d$ is the distance to the nanowire surface. $\epsilon_{1}$ and $\epsilon_{2}$ refer to the dielectric constant of the surroundings and the metal nanowire, respectively.

emitters. In the last section, we fix the operating at $\lambda=525 \mathrm{~nm}$, a wavelength corresponding to the quasi-static regime at the band edge of the dispersion relation.

\section{Results}

In order to discuss the relaxation channels available for coupling the emitter into the plasmonic waveguide, we first introduce the concept of local density of modes in link with the density of modes supported by the metal nanowire.

Metal nanowire: modes density, retarded and quasi-static regimes. Figure 2 represents the dispersion relation of the $T M_{01}$ mode supported by a gold nanowire. Strictly speaking, the stationary mode is defined in absence of dissipation (infinite lifetime), as shown in Fig. 2a. This surface plasmon polariton results from coupling a photon and a surface plasmon so that its dispersion relation closely follows the light line at low frequencies (propagating SPP, retarded regime) and bends towards the quasi-static SPP resonance at larger frequencies. Indeed, in the quasi-static approximation, the SPP resonance condition obeys $\epsilon_{2}(\omega)+\epsilon_{1}=0$, satisfied for $\omega=3.5$ $10^{15} \mathrm{rad} . \mathrm{s}^{-1}(\lambda=525 \mathrm{~nm})$, in the present case. The SPP presents a zero group velocity in this regime (in absence of loss). The density of guided modes $\left(T M_{01}\right)$, proportional to $d k_{z} / d \omega$, is represented in Fig. 2b. It reveals a strong accumulation of modes near $\omega=3.5$ $10^{15} \mathrm{rad} . \mathrm{s}^{-1}$ that defines a common domain with the high-order localized quasi-static modes sustained by a metal nanoparticle. In the following, the quasi-static regime refers to the SPP behaviour near $\lambda=525 \mathrm{~nm}$.
In realistic conditions, the SPP mode must be defined in presence of absorption as shown in Fig. 2c. This defines a quasi mode with a finite lifetime. We observe a similar dispersion curve except near the quasi-static asymptote where a typical back-bending occurs ${ }^{23}$. This area corresponds to a mode lifetime shorter than its oscillation period, or equivalently, a propagation length shorter than the spatial oscillation (see Fig. 2d). The concept of modes is then unavailable. However, this part of the dispersion curve is essential to describe non radiative coupling of a dipolar emitter to a metal nanostructure. We therefore abusively associate the term SPP mode to the whole dispersion curve. The retarded and quasi-static regimes are of great interest to guide (propagating SPP) or confine (quasi-static SPP) light at the nanoscale. The mode propagation length is finally represented in Fig. 2d). As expected, the quasi-static SPP is strongly confined on both the transverse [high $k_{z}$, hence short penetration depth in air $d_{S P P} \sim 1 /\left(k_{z}^{2}-\epsilon_{2} k_{0}^{2}\right)^{1 / 2}$ ] and longitudinal (low propagation length $L_{S P P}$ ) directions. An extremely small modal volume can thus be defined so that efficient coupling to a nearby quantum emitter is expected $^{8,24}$.

Local density of modes, Purcell factor and relaxation channels near a plasmonic wire. The local density of modes (2D-LDOS) near a plasmonic or optical waveguide reads ${ }^{25}$

$$
\Delta \rho^{2 D}\left(\mathbf{r}_{\|}, k_{z}\right)=-\frac{2 k_{z}}{\pi} \operatorname{Im} \operatorname{Tr} \epsilon\left(r_{\|}\right) \Delta \mathbf{G}^{2 D}\left(\mathbf{r}_{\|}, \mathbf{r}_{\|}, k_{z}\right),
$$

where $\Delta \mathrm{G}^{2 D}$ refers to the Green's dyad part associated with the waveguide structure only ( $i$-e excluding its surroundings), $\epsilon\left(r_{\|}\right)$is the dielectric constant at the location $r_{\|}=R+d$ and $k_{z}$ is the wavevector along the nanowire axis. This definition allows to evaluate the density of guided modes (that cancels for $k_{z}=0$ ) but also includes all the scattering and non radiative channels that are excited by a nearby dipolar emitter. This is therefore extremely useful to quantify and describe the dipolar emitter relaxation. Figure $3 \mathrm{a}$ shows the calculated 2D-LDOS near a gold nanowire as a function of its radius at the wavelength $\lambda=800 \mathrm{~nm}$. We observe two resonances corresponding to the $T M_{01}$ and $H E_{11}$ modes, respectively. A strong increase of the $\left(T M_{01}\right) 2 \mathrm{D}$-LDOS appears for small radii that corresponds to a quasi-static-like behavior (different from the quasi-static regime that occurs at a specific wavelength and investigated later). The mode characteristics (wave vector and a)

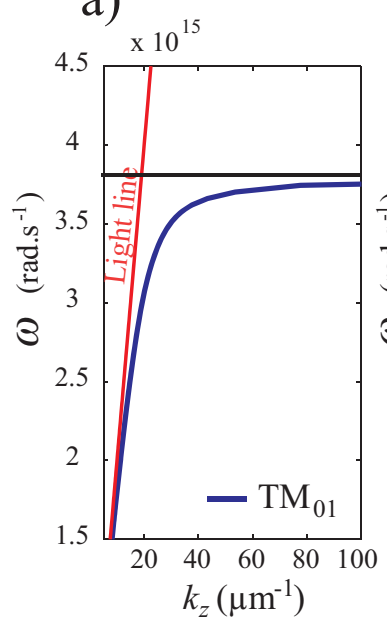

b)

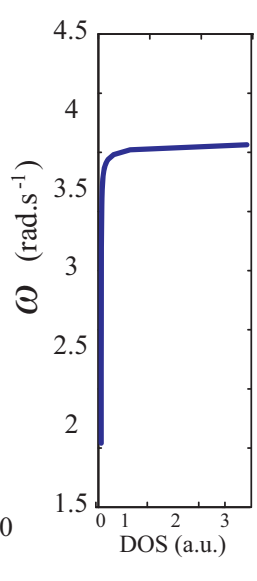

c)

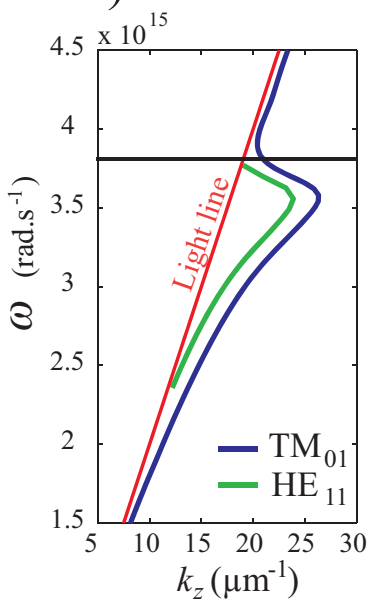

d)

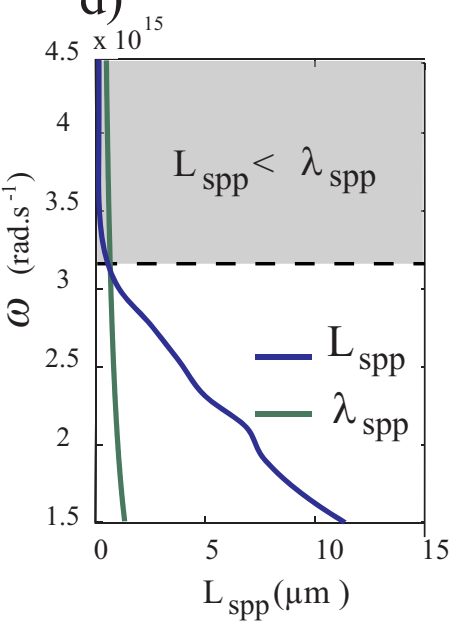

Figure $2 \mid$ (a) Dispersion relation $\omega=f\left(k_{z}\right)$ of a gold nanowire of radius $100 \mathrm{~nm}$ in absence of losses. $k_{z}$ is the propagation constant of the guided

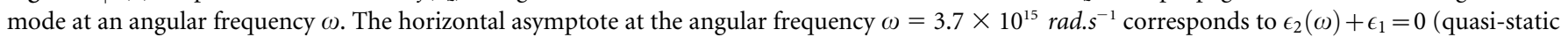
regime). (b) DOS of the $T M_{01}$ mode deduced from $d k_{z} / d \omega$ (in absence of loss). (c) Dispersion relation taking into account losses. (d) Comparison of the $T M_{01}$ mode propagation length $\left(L_{S P P}\right)$ and spatial period $\left(\lambda_{S P P}\right)$. The mode propagation length is shorter than its oscillation period for $\omega>3.10^{15} \mathrm{rad} . \mathrm{s}^{-1}$ (grey area). The gold dielectric constant is taken from Ref. 22 . The dielectric constant of the surrounding medium is $\epsilon_{1}=2.25$. 
a)

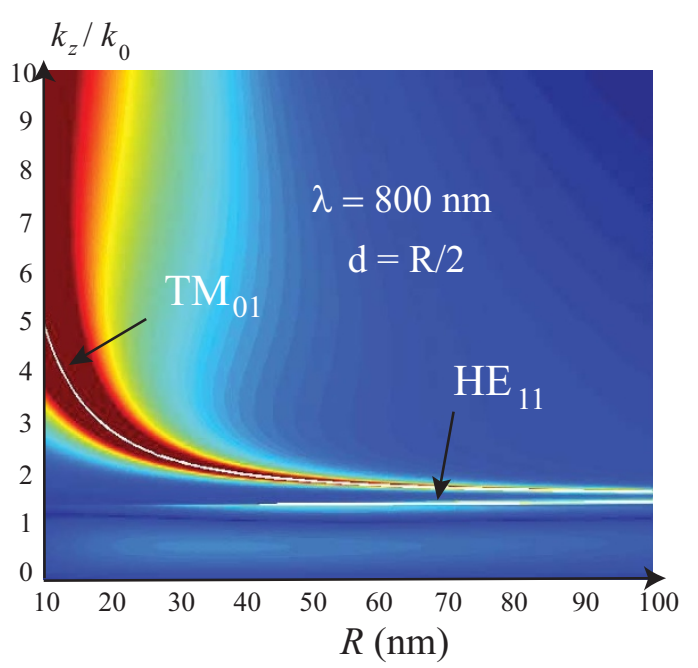

b) $\lambda=800 \mathrm{~nm} \quad d=\mathrm{R}=10 \mathrm{~nm}$

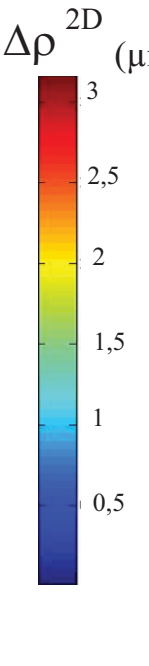

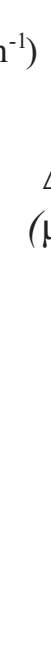
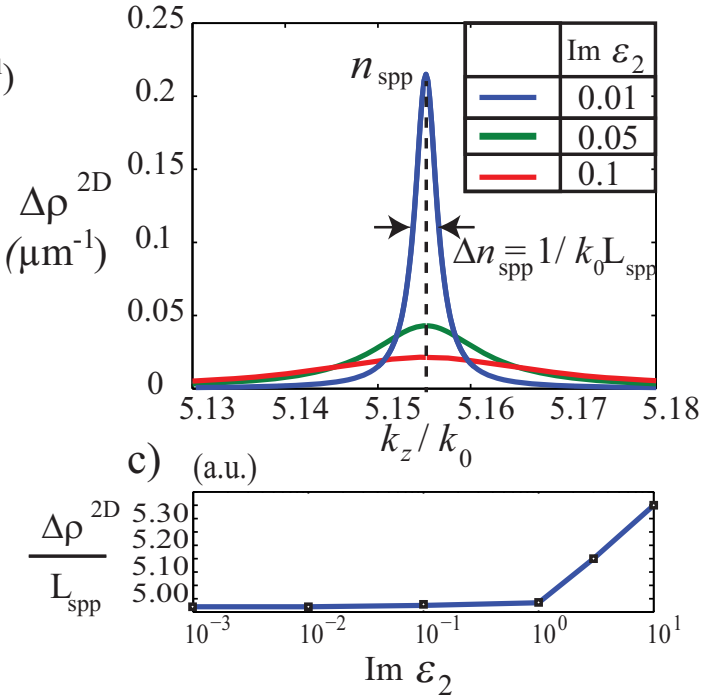

Figure $3 \mid$ (a) 2D-LDOS calculated at distance R/2 from the surface of a gold nanowire as a function of its radius R. The superimposed white lines represents the dispersion relations of the waveguided modes (taking into account the losses. (b) 2D-LDOS as a function of the normalized propagation constant $k_{z} / k_{0}$ for different metal absorption $\operatorname{Im}\left(\epsilon_{2}\right)$. The nanowire radius is fixed at $\mathrm{R}=10 \mathrm{~nm}$. The $2 \mathrm{D}$-LDOS follows a Lorentzian profile peaked at the mode effective index $n_{S P P}$. The FWHM $\Delta n_{S P P}$ is inversely proportional to mode propagation length $L_{S P P}$. (c) Evolution of the ratio $\Delta \rho^{2 D} / L_{S P P}$ as a function of $\operatorname{Im}\left(\epsilon_{2}\right)$.

propagation length) are easily extracted from this formalism ${ }^{25}$. Indeed, for a given radius, the 2D-LDOS presents a Lorentzian profile near SPP resonance. It is peaked at the mode propagation constant and with a full-width-at-half-maximum (FWHM) inversely proportional to the propagation length as shown in Fig. 3b. As expected, the propagation length strongly decreases by increasing the metal absorption $\operatorname{Im}\left(\epsilon_{2}\right)$ and the 2D-LDOS profile flattens. However, the mode effective index $n_{S P P}$ does not depend on the losses. The number of supported modes is given by the area below the 2D-LDOS curve. For a Lorentzian shape, it is proportional to the ratio $\Delta \rho^{2 D} / L_{S P P}$. This ratio is therefore independent on absorption over a very wide range as observed in Fig. 3c. For large losses, the 2D-LDOS does not follows a Lorentzian profile anymore and the concept of mode is then unavailable. In the non-absorbing limit, the 2D-LDOS profile associated to the SPP resonance is a Dirac distribution.

Following Fermi's golden rule, we can now quantify the modification of the fluorescence rate for a point-like dipolar emitter coupled to the nanowire. Indeed, its spontaneous emission is proportional to the $3 \mathrm{D}-\mathrm{LDOS}$ at the emitter position. The 3D-LDOS is simply related to the $k_{z}$-Fourier transform of the 2D-LDOS so that the total decay rate is achieved by integrating the 2D-LDOS on the whole wavevector spectrum (see methods). Physically, the region $\left|k_{z}\right|<n_{1} k_{0}$ corresponds to propagative waves into the medium 1, so-called radiative waves whereas $\left|k_{z}\right|>n_{1} k_{0}$ corresponds to surface waves propagating along the nanowire (including SPP and so-called lossy surfaces waves $)^{27}$. The $2 \mathrm{D}$-LDOS beyond $\left|k_{z}\right|>n_{1} k_{0}$ contributes to the non-radiative rate only but a small amount of radiative waves $\left(\left|k_{z}\right|<n_{1} k_{0}\right)$ could also be absorbed into the metal nanowire. The radiated power is given by the Poynting vector flux. Its computation thanks to the Green's dyad technic involves an integration over the full nanowire volume (or at least on several propagation lengths) ${ }^{28}$, that is a challenging computing task for the infinite wire considered here. Therefore, the radiative rate is evaluated by integrating the $2 \mathrm{D}$ LDOS on the radiative spectrum $\left(\left|k_{z}\right|<n_{1} k_{0}\right)$ and cancelling the metal losses $\left[\operatorname{Im}\left(\epsilon_{2}\right)=0\right]$ and is represented in Fig. 4a. The nonradiative rate is the difference between total and radiative rates and is represented in Fig. 4b. We also represent the results from a quasistatic approximation for comparison ${ }^{29}$.

Let us first discuss the radiative rate behavior (Fig. $4 \mathrm{a}) . \Gamma_{\text {rad }}$ is only weakly modified in presence of the metal nanowire. The quasi-static approximation reproduces very well the distance dependence, except for a dipole parallel to the wire axis (Fig. 4a,iii) but the modification remains low. Indeed, quasi-static approximation considers the dipole induced in the nanowire that cancels along the longitudinal axis ${ }^{29}$. The dependence of radiative decay rate with distance closely resembles to the radiative decay rate near a spherical metal particle ${ }^{30}$ for both radial (Fig. 4a,i) and orthoradial (Fig. 4a,ii) dipoles since the decay rate is governed by the dipole induced in the metal nanoparticle for both geometries.

The non radiative rate is presented in Fig. $4 \mathrm{~b}$. Two main channels contribute to the non radiative coupling ${ }^{26,27}$ : excitation of the guided SPP with a rate $\Gamma_{S P P}$ and lossy surface waves at large $k_{z}$ (see also Fig. $3 \mathrm{a}$ at small radii where high a 2D-LDOS appears for $k_{z}=k_{S P P}$ and above). Although included in the non radiative contribution, the surface plasmon coupled emission exists even for lossless metal and is related to the excitation of a guided mode that can be detected at the nanowire output. This point is discussed in details later. In the case of a realistic absorbing metal, the SPP part contributes to the non radiative channel. SPP absorption originates from the finite conductivity of the metal. It dissipates the energy of the current density associated to the SPP propagation. Therefore, it is referred as Joule (or ohmic) losses. The second dissipation process appearing at large momenta is electron scattering at the lattice impurities. Finally, electron-hole excitation in the metal occurs at very short distances ${ }^{27}$. Note that lossy surface waves describe the dissipated power from both electron scattering and electron-hole excitation. The modelisation of electron-hole excitation would necessitate to include nonlocal effects in the permittivity. This process is negligible for separation distances above $d \simeq 5 \mathrm{~nm}$ considered here ${ }^{27}$.

At very short distances, electron scattering loss dominates $\left(\Gamma_{S P P}\right.$ $\ll \Gamma_{N \text { Rad }}$ ). Above $10 \mathrm{~nm}$, the non radiative channel is dominated by coupling into the propagating SPP (Fig. 4b). We also observe a small contribution of the $H E_{11}$ mode to $\Gamma_{N r a d}$ above $80 \mathrm{~nm}$ in case of 

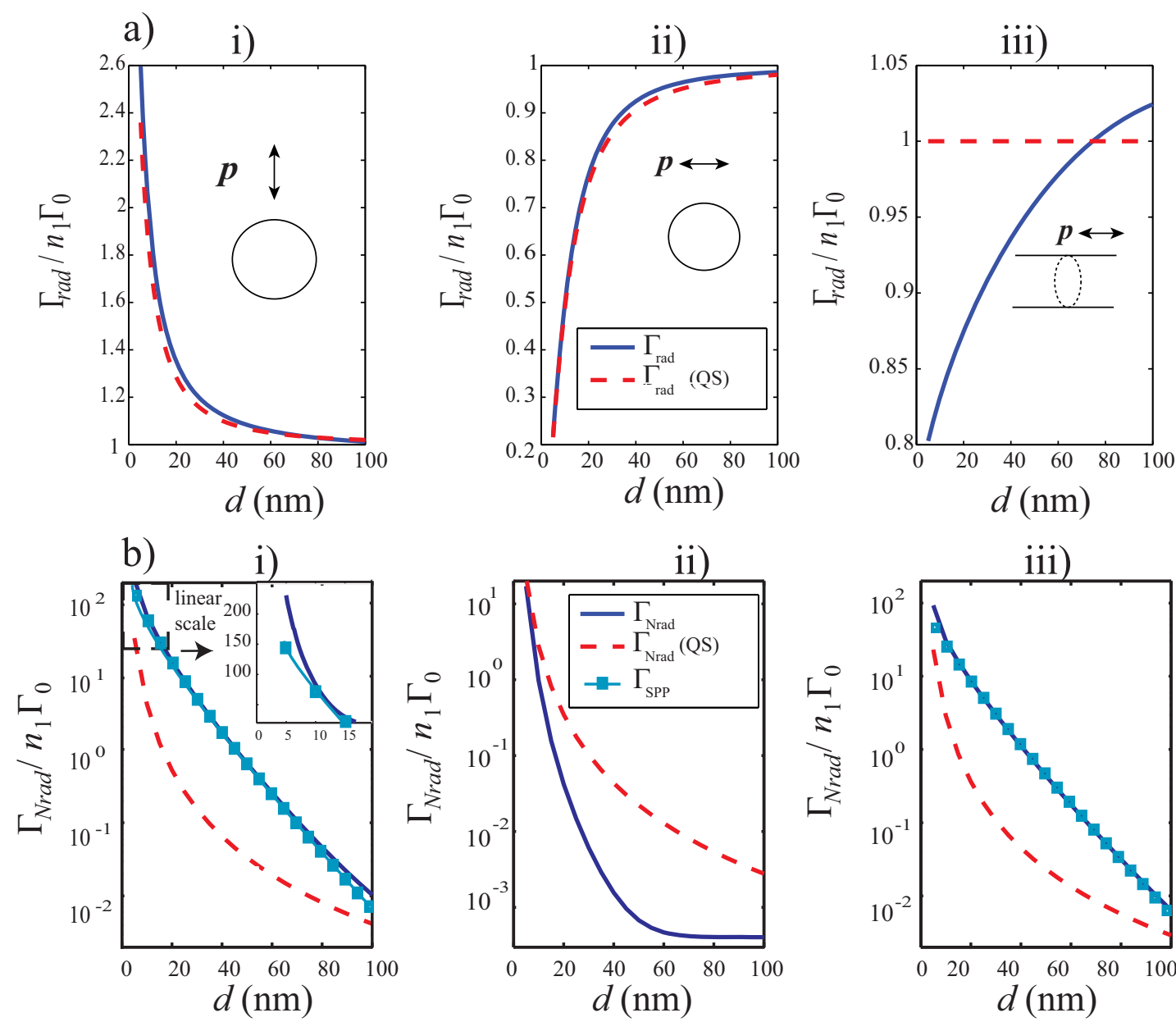

Figure $4 \mid$ Normalized radiative (a) and non-radiative (b) rates of a dipolar emitter placed at a distance $d$ to a gold nanowire for three emitter orientations. The nanowire radius is $R=10 \mathrm{~nm}$ and the emission wavelength is $\lambda=800 \mathrm{~nm}$. The surface plasmon coupled emission contribution to the non radiative rate is also indicated. For orthoradially oriented emitter $\Gamma_{S P P}=0$ [Fig. b,ii)], since guided mode does not possess orthoradial electric component. QS refers to the quasi-static approximation.

radially oriented dipole [see the small difference between $\Gamma_{\text {Nrad }}$ and $\Gamma_{S P P}$ in Fig. $4 \mathrm{~b}(\mathrm{i})$ and Fig. S4 in supplementary information]. For radially (Fig. 4b,i) or longitudinallly (Fig. 4b,iii) oriented emitter, the quasi-static approximation strongly underestimates the non radiative rate since it does not include SPP contribution. In the case of an orthoradial emitter, the SPP contribution is null and the quasistatic approximation is slightly better (Fig. 4b,ii).

The surface plasmon coupled emission finally expresses as ${ }^{26}$

$$
\frac{\Gamma_{S P P}}{n_{1} \Gamma_{0}}=\frac{3 \pi \lambda}{4 n_{1}^{3} k_{S P P}} \frac{\Delta \rho_{\mathbf{u}}^{2 D}\left(\mathbf{r}_{\|}, k_{S P P}\right)}{L_{S P P}}
$$

where both the forward and backward propagation modes are included. This expression extends the definition of the Purcell factor to a guided mode picture making a useful link with cavity quantum electrodynamics. As expected, the coupling rate depends on the overlap with the mode profile (represented by the density of guided mode $\Delta \rho^{2 D}$ ) so that it decreases with the emitter-nanowire distance (penetration depth in air at $\lambda=800 \mathrm{~nm}: d_{S P P} \sim 26 \mathrm{~nm}$ ). The $1 / L_{S P P}$ dependence is more surprising at first glance. Close inspection of the expression clarifies this point. This Purcell factor (Eq. 2) is constant on a large absorption range, since the ratio $\Delta \rho^{2 D} / L_{S P P}$ determines the number of modes and does not depend on the losses (see Fig. 3b,c). This implies that an emitter couples to the waveguide independently on the following dissipations: Joule losses or propagation up to the waveguide output ${ }^{26}$. Obviously, lower absorption is preferred to achieve a sufficient signal level at the waveguide output. This original result can also be understood from the expression of the Purcell factor

$$
F_{p}=\frac{\Gamma_{S P P}}{n_{1} \Gamma_{0}}=\frac{3}{4 \pi^{2}}\left(\frac{\lambda}{n_{1}}\right)^{3} \frac{Q}{V_{e f f}},
$$

where $Q$ and $V_{\text {eff }}$ refer to the mode quality factor and effective volume, respectively. Here, the mode quality factor is $Q=k_{S P P} /$ $\Delta k_{S P P}=n_{S P P} k_{0} L_{S P P}$ (typically $Q=12$ for $R=10 \mathrm{~nm}$ and $Q=62$ for $R=100 \mathrm{~nm}$, see supplementary information). The mode volume is estimated as follows. The mode confinement in the transverse plane is characterized by an effective surface $A_{\text {eff }}$ that we explicit later on. Due to the finite propagation length $L_{S P P}$ in the longitudinal direction, the mode volume varies as $V_{\text {eff }} \sim A_{\text {eff }} \times L_{S P P}$. Finally, the Purcell factor $F_{p} \propto Q / V_{\text {eff }} \propto A_{\text {eff }}$ does not depend on $L_{S P P}$. Identifying expressions (2) and (3), we can deduce the mode volume of the guided SPP. For this, we assume a randomly oriented emitter located at the nanowire surface (see also Ref. 24). Since the Purcell factor does not depend on Joule losses, the mode area is computed using the lossless coupling rate description (see supplementary information). We represent in Fig. 5a the guided SPP mode volume for nanowire radius varying from $10 \mathrm{~nm}$ to $100 \mathrm{~nm}$. Its is significantly subwavelength demonstrating the ability of surface plasmon to confine light at the nanoscale. It is however worthwhile to note that the mode confinement along the longitudinal direction is associated to the finite propagation length $L_{S P P}$. Since it is compensated by 


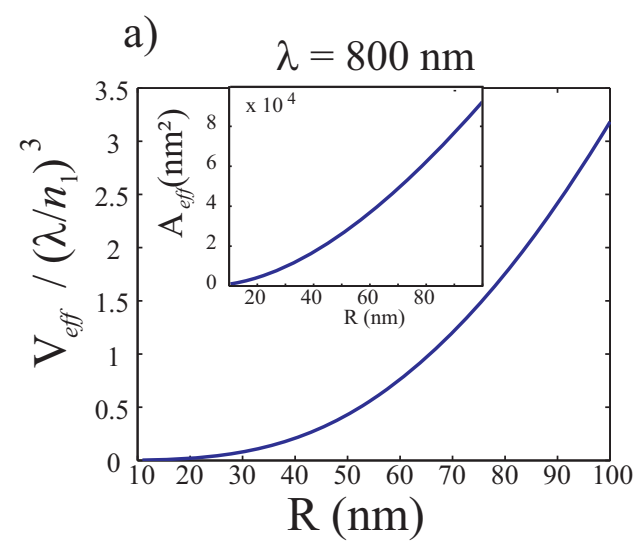

b)

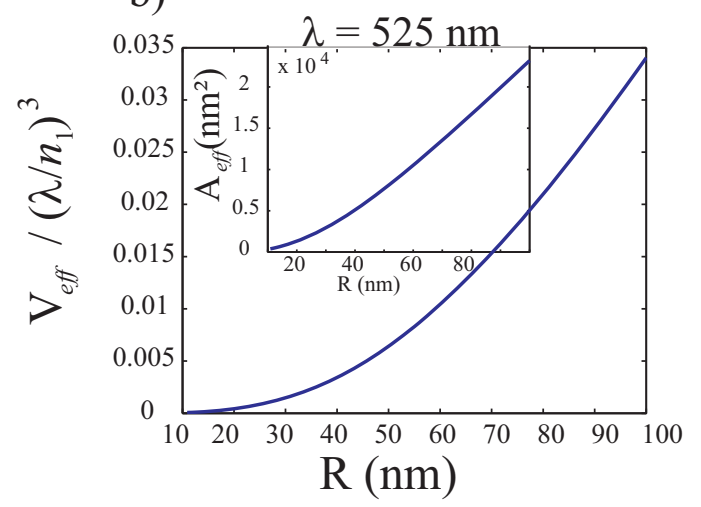

Figure $5 \mid$ Normalized effective volume and area (insets) as a function of nanowire radius at (a) $\lambda=800 \mathrm{~nm}$ and (b) $\lambda=525 \mathrm{~nm}$. the mode quality factor $Q$ in the Purcell factor, the only parameter of interest is the mode effective area represented in the inset of Fig. 5a. As a partial conclusion, we stress that the plasmonic Purcell factor has to be manipulated with care and cannot be used as a figure of merit of the coupled system without an additionnal analysis of losses. Figure $5 \mathrm{~b}$ presents the mode confinement at $\lambda=525 \mathrm{~nm}$ for comparison and will be discussed in the last paragraph.

Dipole-SPP coupling efficiency and SPP mediated dipole-dipole coupling. Thus far we investigated in details the various relaxations channels for a dipolar emitter coupled to a metal nanowire. Finally, a key parameter is the $\beta$-factor that quantifies the coupling efficiency into the guided SPP as compared to all available channels. $\beta=\Gamma_{\text {SPP }} /$ $\left(\Gamma_{\text {Nrad }}+\Gamma_{\text {rad }}\right)$ is represented in Fig. 6a. At very short distances electron scattering loss dominates. At long distances the plasmonic decay rate becomes negligible compared to the radiative rate. But for emitter-nanowire distances in the 10-30 $\mathrm{nm}$ range, a high coupling efficiency above $85 \%$ is achieved for both longitudinal and radial orientations. As discussed previously, surface plasmon coupled emission $\Gamma_{S P P}$ does not depend on Joule losses. Nevertheless, low loss systems such as crystalline nanowire are evidently preferred. We recently demonstrated that the plasmon coupling rate to a pentatwinned crystalline silver nanowire is optimized near the nanowire edges $^{12,26}$. This means that the plasmon coupling rate $\Gamma_{S P P}$ is similar, or even better, to the case studied here. Additionally, crystalline nanowire presents lower losses $\operatorname{rate}^{10} \Gamma_{N R}$ leading to higher $\beta$ factor and signal level at the plasmonic waveguide output.

Since excellent surface plasmon coupled emission is achieved, we now consider two coupled emitters placed near the nanowire at distance $\Delta z$ from each other. The coupling rate $\Gamma_{12}$ between two radial emitters is represented in Fig. $6 \mathrm{~b}$ as a function of the emitter's separation distance. If a donor emitter in its excited state is weakly coupled to an acceptor emitter in its ground state, $\Gamma_{12}$ refers to the

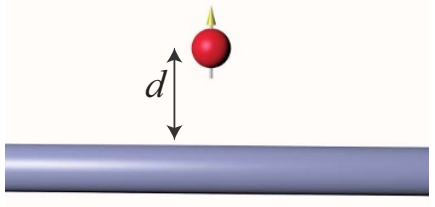

a)

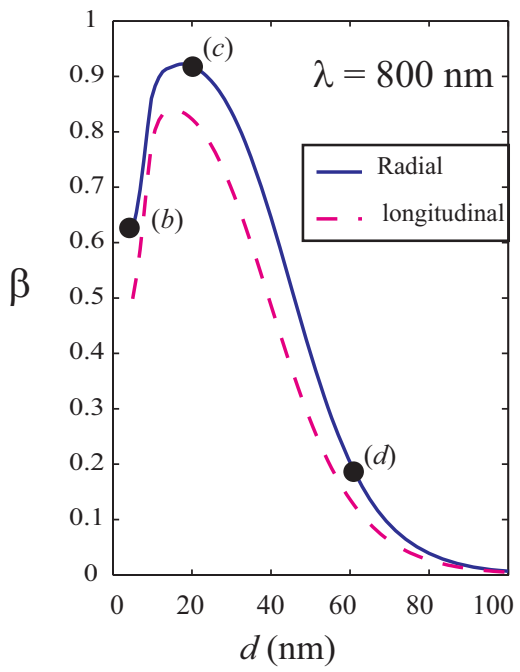

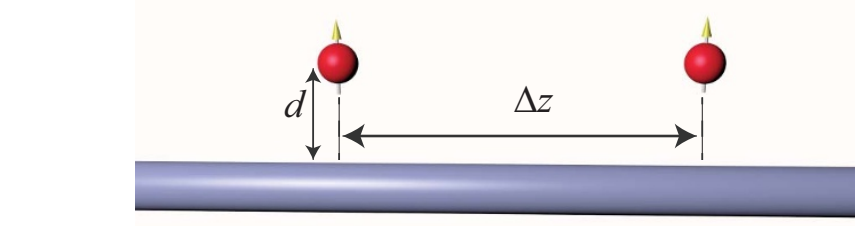
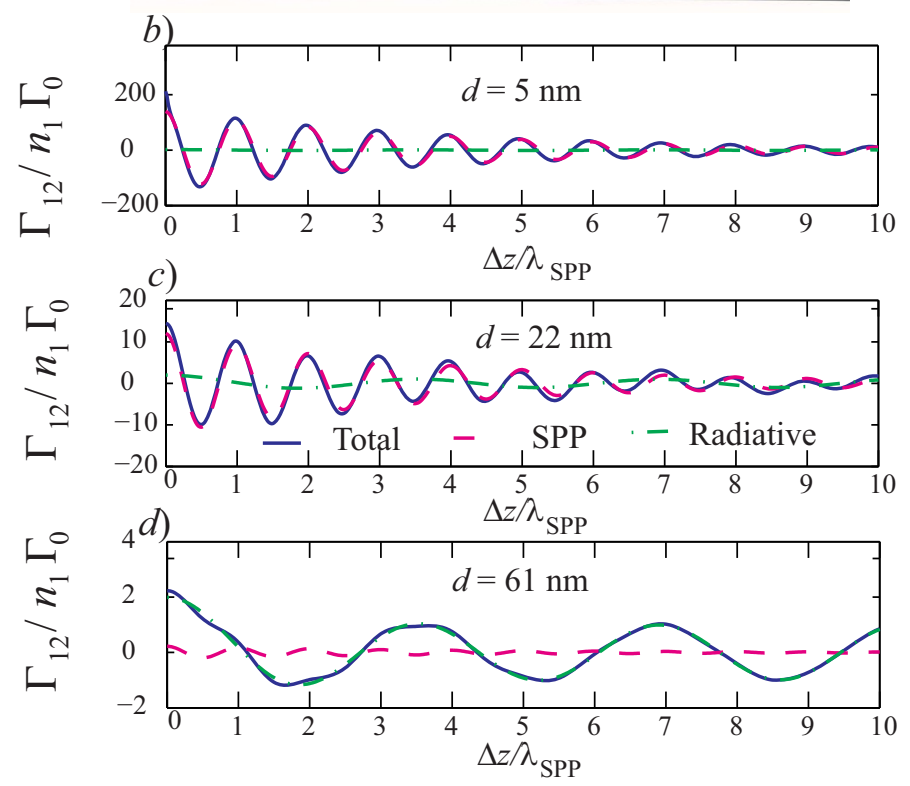

Figure 6 (a) $\beta$-factor near a $R=10 \mathrm{~nm}$ gold nanowire at $\lambda=800 \mathrm{~nm}$ as a function of the emitter-nanowire distance $d$. (b-d) Collective decay rate $\Gamma_{12}$ for two radially oriented dipolar emitters as a function of their separation distance $\Delta z$ for three distances $d$ to the nanowire and three emitters orientations. The dotted red and dashed-dotted green curves refer to the SPP and radiative contributions, respectively. Distance is normalized with respect to the SPP wavelength. 
coupling rate between the two emitters. This weak coupling leads to an irreversible resonant energy transfer from the donor to the acceptor $^{31-34}$. Differently, if the two emitters are strongly coupled, the system cannot be described from isolated dipolar emitters in their ground $(g)$ or excited $(e)$ state. One rather have to introduce the coupled basis $|e e>| g g>,, \mid+>=(|e g>+| g e>) / \sqrt{(2)}$ and $\mid->=(|e g>-| g e>) / \sqrt{(2)}$ for identical emitters. The two entangled states $\mid+>$ and $\mid->$ are of particular interest since they do not possess classical counterparts. $\Gamma_{12}$ is the so-called cooperative decay rate of the coupled system and $\Gamma_{11} \pm \Gamma_{12}$ refer to the decay rates of these coupled states. Positive and negative cooperative rates $\Gamma_{12}$ correspond to superradiance and subradiance of the two coupled emitters, respectively (note that the physical decay rates $\Gamma_{11} \pm \Gamma_{12}$ are always positives). We represent in Fig. $6 \mathrm{~b}$ the cooperative decay rate. We also evaluated the radiative and SPP contributions to the collective emission. The highest collective decay rate (Fig. 6b) corresponds to the collective excitation of guided SPP but also lossy waves. The radiative contribution to the collective relaxation is negligible. Therefore the useful part of the collective decay rate is the collective surface plasmon coupled emission, achieved for the highest $\beta$-factor, that is for $d=22 \mathrm{~nm}$ in the present case (Fig. 6a,c). For emitters close to the nanowire the coupling rate $\Gamma_{12}$ presents an oscillatory behavior with a spatial period corresponding to the SPP wavelength (Fig. 6b,c). For larger separation distances, the cooperative decay rate presents a more complex behaviour due to a beating between free-space direct emission and the surface plasmon coupled emission (see Fig. $6 \mathrm{~d}$ and S8).

Quasi-static regime. In this last section, we investigate the surface plasmon coupled emission at the band edge of the dispersion relation, corresponding to the quasi-static behavior of the SPP (see
Fig. 2). Indeed, the mode group velocity strongly slows down in this spectral range, and we therefore expect important light-matter interaction enhancement in this regime ${ }^{19,35-37}$.

Radiative and non radiative rates are calculated in Fig. 7 for an emission wavelength $\lambda=525 \mathrm{~nm}$. The radiative rate is significantly enhanced near the surface plasmon resonance (Fig. 7a). As expected, the quasi-static approximation better describes the non radiative coupling as compared to the retarded regime (compare Figs. $7 \mathrm{~b}$ and $4 \mathrm{~b}$ ). The non radiative coupling is dominated by electron scattering. For comparison purpose, we also represent the SPP contribution to the non radiative rate, even if the definition of the plasmonic mode becomes questionable in this regime (cf. Fig. 2). Due to strong losses, the 2D-LDOS no more follows a lorentzian profile as in Fig. 3b). Therefore, we estimate the SPP contribution using a lossless guide description since we demonstrated earlier that it correctly describes the coupling rate to the guided mode ${ }^{26}$. This is however an extrapolation from the propagating regime situation. It is nonetheless helpful for a qualitative discussion.

The coupling rate into the SPP decreases very fast as a function of the distance (Fig. 7b). This abrupt decay reveals the strong mode confinement at the metal surface. We deduce from the slope the penetration of the mode in air to $d_{S P P}=9 \mathrm{~nm}$. This is confirmed by the calculated mode effective volume or area represented in Fig. 5b. In this quasi-static regime, the SPP mode volume reaches extremely low value $V_{\text {eff }} \sim 10^{-4}\left(\lambda / n_{1}\right)^{3}$ similar to the localized SPP configuration $^{24,38}$. Nevertheless, strong losses limits the maximum achievable $\beta$-factor to $\beta \approx 30 \%$ at $d=15 \mathrm{~nm}$ (not shown).

Finally, Fig. 8 presents the cooperative decay rate for two quantum emitters near the metallic nanowire at the emission wavelength $\lambda=$ $525 \mathrm{~nm}$. Due to strong losses, SPP coupling does not exist anymore. We rather observe a low collective decay rate, governed by radiative a)

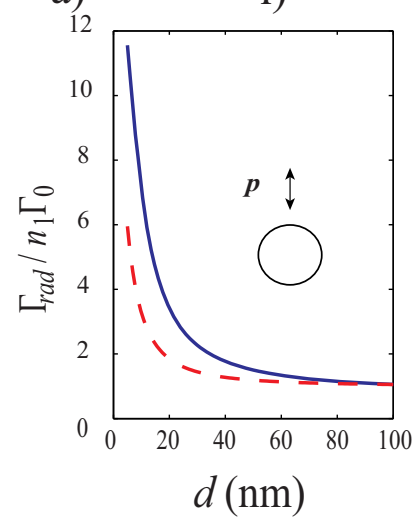

b)

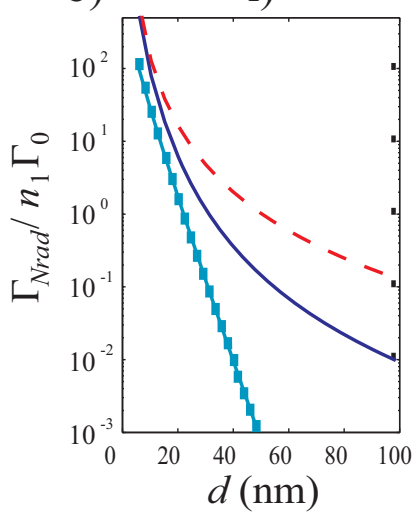

ii)

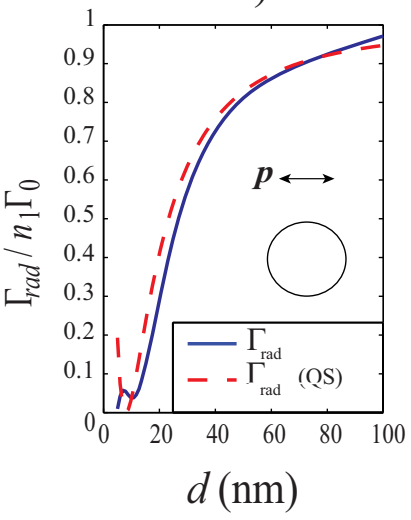

ii)

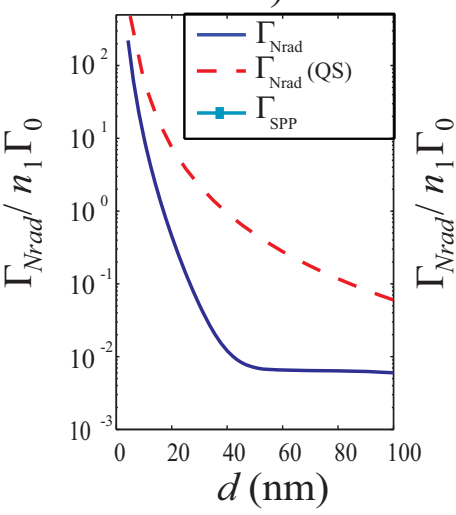

iii)

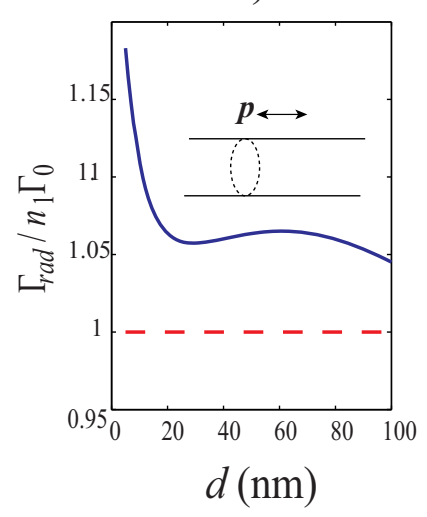

iii)

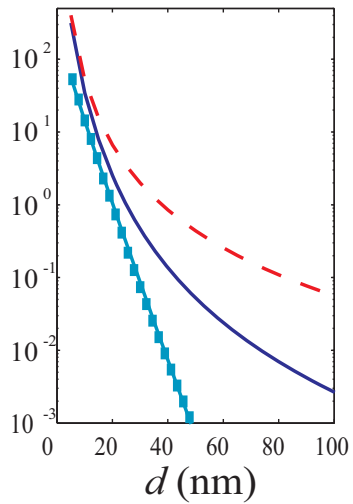

Figure $7 \mid$ Same as Fig. 4 for the emission wavelength $\lambda=525 \mathrm{~nm}$. 


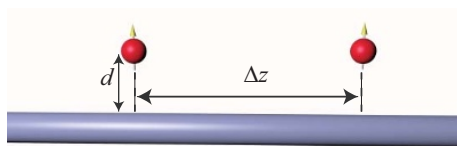

radial orientation

$d=11 \mathrm{~nm}$

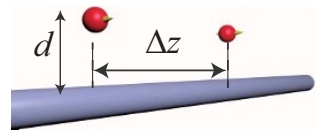

orthoradial orientation

$d=11 \mathrm{~nm}$

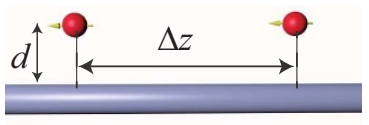

longitudinal orientation $d=11 \mathrm{~nm}$
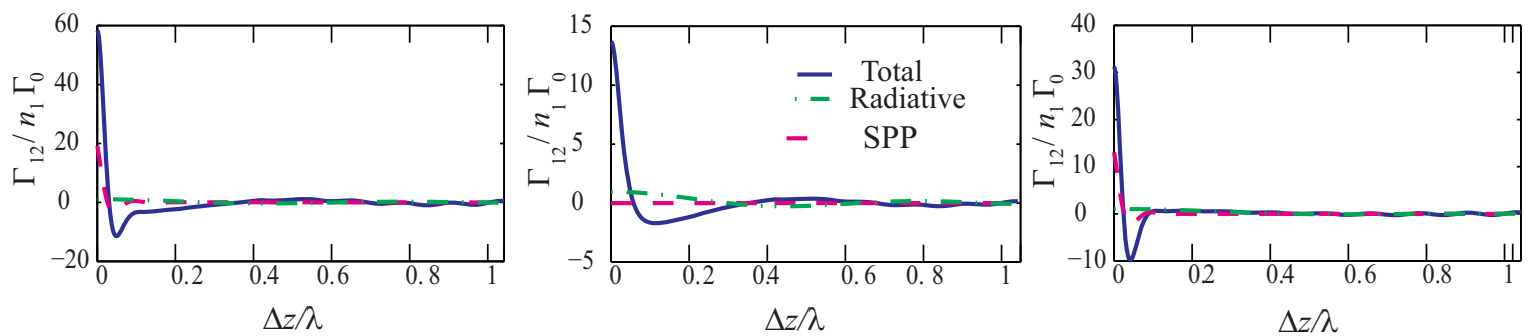

Figure $8 \mid$ Collective decay rate $\Gamma_{12}$ for two dipolar emitters as a function of their separation distance $\Delta z$ for three emitters orientations. The dotted red curves refer to the radiative contribution. The emitter is $11 \mathrm{~nm}$ away from the nanowire surface. Distance is normalized with respect to the free-space wavelength.

contribution since only direct free-space coupling occurs. Therefore the pseudo-periodic behavior of the cooperative decay rate follows the free-space wavelength. It is moreover strongly damped due to high losses in this regime.

\section{Discussion}

A 1D plasmonic waveguide efficiently promotes light-matter interaction at the nanoscale. Although the SPP presents a modest quality factor (of the order of 10 to 100), they possess extremely low modal area (or volume) that are not diffraction limited and are thus very promising for enhancing single atom/single photon process. In the retarded regime, we demonstrated modal volume typically ten times below the diffraction limit and $\beta$ factor up to $90 \%$ but that could be still increased if crystalline nanostructures are involved. In this work, we also clarified the effect of Joule losses and proposed a simple physical understanding of the coupling mechanism in surface plasmon coupled emission. We expect that this would be also helpful in defining strong coupling regime conditions ${ }^{39}$.

Finally, we discussed the cooperative behavior of two coupled quantum emitters and demonstrated an efficient collective excitation of guided SPPs. It has been recently proposed to entangle two remotes qubits ${ }^{18,19}$ or to realize nano-optical logical gates ${ }^{20}$ by controlling sub/superradiance. This regime is also of strong interest in the context of superradiant lasers (also called bad cavity lasers) presenting high spectral purity ${ }^{40,41}$. Due to the poor quality factor of SPP resonances, we envision a fruitful analogy for designing plasmon nanolasers ${ }^{42-44}$. Indeed, the collective relaxation of several identical emitters in a poor cavity can lead to a coherent emission with extremely narrow spectral width ${ }^{40,41}$. Practically, the limit linewidth of a single mode laser writes ${ }^{45}$

$$
\Delta v=\frac{h v}{4 \pi} \frac{N_{s p}}{P_{\text {out }}}\left(\frac{\Gamma_{0} \kappa}{\Gamma_{0}+\kappa / 2}\right)^{2}
$$

where $\kappa$ is the cold-cavity loss rate, $P_{\text {out }}$ the laser output power, and $N_{s p}=N_{e} /\left(N_{e}-N_{g}\right)$ the degree of population inversion. In a good cavity regime $(\kappa \ll 2 \Gamma)$, the laser spectral width is governed by cavity loss rate. It follows the Schawlow-Townes limit $\Delta v=\frac{h v}{4 \pi} \frac{\kappa^{2}}{P_{\text {out }}}$. However, as far as SPP amplication is concerned, one as to considered the bad cavity regime $(\kappa \gg 2 \Gamma): \Delta v=\frac{h v}{4 \pi} \frac{N_{s p} 4 \Gamma_{0}^{2}}{P_{\text {out }}}$. This defines the superradiant laser for which the electromagnetic coherence originates from the collective behaviour of the gain medium instead of the cavity feedback. Here, the cavity mode is only necessary to collectively couple the atoms forming the gain medium. The laser linewidth is then limited by the gain medium and could be largely below the Schawlow-Townes limit ${ }^{40,41}$. Note also that a partial population inversion $N_{s p}$ degrades the linewidth lower limit. This has to be taken into account when describing SPP nanolasers since non radiative energy transfer to the metal limits the population inversion ${ }^{46}$. Since the SPP guided mode presents a rather low quality factor but mediates efficiently cooperative relaxation, we expect a fruitful analogy with superradiant lasers to achieve a coherent SPP nanosource.

Last, we investigated the quasi-static regime and demonstrated SPP confinement down to $10^{-4}\left(\lambda / n_{1}\right)^{3}$ but very low surface plasmon coupled emission due to critically important losses. Although limited to plasmonics nanowires in this work, the rich physics underlying the coupling between single photon source(s) and lossy materials is very general and remains valid for arbitrary geometries as well as metamaterials. Additionally, the extreme mode confinement observed at the band-edge of the dispersion relation is closely related to localized surface plasmon and create a bridge with cavityless quantum electrodynamics.

\section{Methods}

Dispersion curve. The dispersion curve of the metal nanowire is achieved by solving the dispersion relation of the $T M_{01}$ and $H E_{11}$ modes with Davidenko's algorithm in the complex plane $e^{47}$. This leads to complex propagation constant solutions $\tilde{k}_{S P P}(\omega)=k_{S P P}+i k_{S P P}$. The mode propagation length obeys $L_{S P P}=1 / 2 k^{\prime \prime}{ }_{S P P}$. Davidenko's algorithm is an efficient method to find complex root, including leaky modes (mode effective index below the surrounding optical index). In the present case, we observe that the $H E_{11}$ mode is not cut-off but its effective index approaches that of the surrounding optical index for small nanowire in agreement with the 2DLDOS calculation (see Fig. 3) and also with the asymptotic expansion derived in ref. 8 (see also supplementary information).

Green's dyad. The 2D-Green's dyad is numerically computed discretizing the nanowire cross-section with rectangular meshes as detailed in ref. 48. Meshes sizes varies from $0.5 \mathrm{~nm}$ to $10 \mathrm{~nm}$ for larger radii. Importantly, the Green's dyad variation inside the mesh is exactly included, ensuring accurate evaluation. Arbitrary waveguide profile eventually supported on a wafer, are easily treated with this method $^{26}$. Symmetrical properties of the Green's tensor are used to limit the numerical evaluation of $\mathrm{G}^{2 D}$ on the $k_{z} \geq 0$ range. Indeed, $\mathrm{G}^{2 D}\left(\mathbf{r}_{1 / /}, \mathbf{r}_{2 / /},-k_{z}\right)=$ $\left[\mathrm{G}^{2 D}\left(\mathbf{r}_{2 / /}, \mathbf{r}_{1 / /}, k_{z}\right)\right]^{T}$ (see supplementary information). The behaviour of $\mathrm{G}^{2 D}$ near SPP resonance is also discussed in the supplementary information (Fig. S6).

Decay rates. The dipolar total decay rate expresses $\Gamma_{t o t}(x, y) / n_{1} \Gamma_{0}=-3 / n_{1} k_{0}$ $\int_{-\infty}^{+\infty} \operatorname{Im}\left[\mathbf{u} \cdot \mathbf{G}^{2 D}\left(\mathbf{r}_{0 / /}, \mathbf{r}_{0 / /}, k_{z}\right) \cdot \mathbf{u}\right] d k_{z}$ where $\mathbf{r}_{\mathbf{0} / /}=(x, y)$ refers to the emitter position in the transverse plane and $\mathbf{p}=p \mathbf{u}$ is the dipolar transition moment. The collective decay rate of two coupled dipoles $\left(\mathbf{p}_{1}=p_{1} \mathbf{u}_{1}, \mathbf{p}_{2}=p_{2} \mathbf{u}_{2}\right)$ writes $\Gamma_{12} / n_{1} \Gamma_{0}=-3 / n_{1} k_{0}$ $\int_{-\infty}^{+\infty} \operatorname{Im}\left[e^{i k_{z} \Delta z} \mathbf{u}_{1} \cdot \mathbf{G}^{2 D}\left(\mathbf{r}_{1 / /}, \mathbf{r}_{2 / /}, k_{z}\right) \cdot \mathbf{u}_{2}\right] d k_{z}$ with $\mathbf{r}_{1 / /}$ and $\mathbf{r}_{2 / /}$ the dipole positions in the transverse plane and $\Delta z=z_{1}-z_{2}$ their separation distance. Radiative rates $\Gamma_{\text {rad }}$ 
and $\Gamma_{12}^{r a d}$ are obtained limiting the integration on the radiative waves $\left(\left|k_{z}\right|<n_{1} k_{0}\right)$ and cancelling the metal losses $\left[\operatorname{Im}\left(\epsilon_{2}\right)=0\right]$. SPP contributions $\Gamma_{S P P}$ and $\Gamma_{12}^{S P P}$ are achieved keeping the SPP resonance only into the integration. Thanks to the Lorentzian shape of the resonance, analytical expressions are available (see Eq. 2 and supplementary information)

Relaxation channels in lossless or quasi-static limits. The Purcell factor near a nonabsorbing waveguide is given by ${ }^{21,49}$

$$
\frac{\Gamma_{p l, \mathbf{u}}}{\Gamma_{0}}=\frac{3 \pi c \epsilon_{0}\left|E_{\mathbf{u}}(R+d)\right|^{2}}{k_{0 A_{\infty}}^{2}(\mathbf{E} \times \mathbf{H} *) \cdot \mathbf{z} \cdot d A},
$$

where $(\mathrm{E}, \mathrm{H})$ is the modal electromagnetic field, * refers to the conjugated form and $\mathbf{u}$ is the emitter orientation. For a circular cross-section, it is analytical (see supplementary information).

In the quasi-static limit, radiative and non radiative rates expressions are given in ref. 29 .

1. Vahala, K. J. Optical microcavities. Nature 424, 839-846 (2003).

2. Birowosuto, M. et al. Fast purcell-enhanced single photon source in 1,550-nm telecom band from a resonant quantum dot-cavity coupling. Sci. Rep. 2, 321 (2012).

3. Nayak, K. P. \& Hakuta, K. Single atoms on an optical nanofibre. New J. Phys. 10, 053003 (2008)

4. Jacob, Z. Quantum plasmonics. MRS Bulletin 37, 761-767 (2012).

5. Kolesov, R. et al. Wave-particle duality of single surface plasmon polaritons. Nat. Phys. 5, 470-474 (2009).

6. Mollet, O., Huant, S., Dantelle, G., Gacoin, T. \& Drezet, A. Quantum plasmonics: second-order coherence of surface plasmons launched by quantum emitters into a metallic film. Phys. Rev. B 86, 045401 (2012).

7. Noskov, R., Belov, P. \& Kivshar, Y. Oscillons, solitons, and domain walls in arrays of nonlinear plasmonic nanoparticles. Sci. Rep. 2, 873 (2012).

8. Chang, D., Sörensen, A., Hemmer, P. \& Lukin, M. Strong coupling of single emitters to surface plasmons. Phys. Rev. B 76, 35420 (2007).

9. Wei, $\mathrm{H} . \& \mathrm{Xu}, \mathrm{H}$. Nanowire-based plasmonic waveguides and devices for integrated nanophotonic circuits. Nanophotonics 1, 155-169 (2012).

10. Ditlbacher, H. et al. Silver nanowires as surface plasmon resonators. Phys. Rev. Lett. 95, 257403 (2005).

11. Laroche, T., Vial, A. \& Roussey, M. Crystalline structure's influence on the nearfield optical properties of single plasmonic nanowires. Appl. Phys. Lett. 91, 123101 (2007)

12. Song, M. et al. Imaging symmetry-selected corner plasmon modes in pentatwinned crystalline Ag nanowires. ACS Nano 5, 5874-5880 (2011).

13. Huck, A., Kumar, S., Shakoor, A. \& Andersen, U. L. Controlled coupling of a single nitrogen-vacancy center to a silver nanowire. Phys. Rev. Lett. 106, 096801 (2009).

14. Gruber, C., Kusar, P., Hohenau, A. \& Krenn, J. R. Controlled addressing of quantum dots by nanowire plasmons. Appl. Phys. Lett. 100, 221102 (2012).

15. Ropp, C. et al. Nanoscale imaging and spontaneous emission control with a single nano-positioned quantum dot. Nat. Commun. 4, 1447 (2013).

16. Chang, D., Sörensen, A., Demler, E. \& Lukin, M. A single-photon transistor using nanoscale surface plasmons. Nat. Phy. 3, 807-812 (2007).

17. Martin-Cano, D., Martin-Moreno, L., Garcia-Vidal, F. \& Moreno, E. Resonance energy transfer and superrradiance mediated by plasmonic nanowaveguides. Nano Lett. 10, 3129-3134 (2010).

18. Gonzalez-Tudela, A. et al. Entanglement of two qubits mediated by onedimensional plasmonic waveguides. Phys. Rev. Lett. 106, 020501 (2011).

19. Chen, G.-Y., Lamber, N., Chou, C.-H., Chen, Y.-N. \& Nori, F. Surface plasmons in a metal nanowire coupled to colloidal quantum dots: Scattering properties and quantum entanglement. Phys. Rev. B 84, 045310 (2011).

20. Dzsotjan, D., Sorensen, A. S. \& Fleischhauer, M. Quantum emitters coupled to surface plasmons of a nanowire: A Green's function approach. Phys. Rev. B 82, 075427 (2010)

21. Chen, Y., Nielsen, T. R., Gregersen, N., Lodahl, P. \& Mork, J. Finite-element modeling of spontaneous emission of a quantum emitter at nanoscale proximity to plasmonic waveguides. Phys. Rev. B 81, 125431 (2010).

22. Johnson, P. \& Christy, R. Optical constants of the noble metals. Phys. Rev. B 6 , 4370-4379 (1972).

23. Archambault, A., Teperik, T. V., Marquier, F. \& Greffet, J. J. Surface plasmon Fourier optics. Phys. Rev. B 79, 195414 (2009).

24. Derom, S., Vincent, R., Bouhelier, A. \& Colas des Francs, G. Resonance quality, radiative/ohmic losses and modal volume of Mie plasmons. EPL 98, 47008 (2012).

25. Colas des Francs, G. et al. Integrated plasmonic waveguides: a mode solver based on density of states formulation. Phys. Rev. B 80, 115419 (2009).

26. Barthes, J., Colas des Francs, G., Bouhelier, A., Weeber, J.-C. \& Dereux, A. Purcell factor for a point-like dipolar emitter coupling to a $2 \mathrm{D}$-plasmonic waveguide. Phys. Rev. B 84, 073403 (2011).

27. Ford, G. W. \& Weber, W. H. Electromagnetic interactions of molecules with metal surfaces. Phys. Rep. 113, 195-287 (1984).
28. Colas des Francs, G., Girard, C., Juan, M. \& Dereux, A. Energy transfer in nearfield optics. J. Chem. Phys. 123, 174709 (2005).

29. Klimov, V. V., Lebedev, N. \& Ducloy, M. Spontaneous emission rate of an excited atom placed near a nanofiber. Phys. Rev. A 69, 013812 (2004).

30. Colas des Francs, G. et al. Fluorescence relaxation in the near-field of a mesoscopic metallic particle: distance dependence and role of plasmon modes. Opt. Exp. 16, 17654-17666 (2008).

31. Andrew, P. \& Barnes, W. L. Förster energy transfer in an optical microcavity. Science 290, 785-788 (2000).

32. Colas des Francs, G., Girard, C. \& Martin, O. Fluorescent resonant energy transfer in the optical near-field. Phys. Rev. A 67, 53805 (2003).

33. Klimov, V., Sekatskii, S. K. \& Dietler, G. Coherent fluorescence resonance energy transfer between two dipoles: Full quantum electrodynamics approach. J. Mod. Opt. 51, 1919-1947 (2004).

34. Le Kien, F., Gupta, S. D., Nayak, K. \& Hakuta, K. Nanofiber-mediated radiative transfer between two distant atoms. Phys. Rev. A 72, 063815 (2005).

35. Chen, Y. N., Chen, G. Y., Chuu, D. S. \& Brandes, T. Quantum-dot exciton dynamics with a surface plasmon: Band-edge quantum optics. Phys. Rev. A 79, 033815 (2009).

36. He, S., He, Y. \& Jin, Y. Revealing the truth about 'trapped rainbow' storage of light in metamaterials. Sci. Rep. 2, 583 doi:10.1038/srep00583 (2012).

37. Hu, H., Ji, D., Zeng, X., Liu, K. \& Gan, Q. Rainbow trapping in hyperbolic metamaterial waveguide. Sci. Rep. 3, 1249 doi:10.1038/srep01249 (2013).

38. Sauvan, C., Hugonin, J.-P., Maksymov, S. \& Lalanne, P. Theory of the Spontaneous Optical Emission of Nanosize Photonic and Plasmon Resonators. Phys. Rev. Lett. 110, 237401 (2013).

39. Hümmer, T., García-Vidal, F. J., Martín-Moreno, L. \& Zueco, D. Weak and strong coupling regimes in plasmonic QED. Phys. Rev. B 87, 115419 (2013).

40. Haake, F., Kolobov, M., Fabre, C., Giacobino, E. \& Reynaud, S. Superradiant laser. Phys. Rev. Lett. 71, 995-998 (1993).

41. Bohnet, J. G. et al. A steady-state superradiant laser with less than one intracavity photon. Nature 484, 78-81 (2012).

42. Grandidier, J. et al. Gain assisted propagation in a plasmonic waveguide at telecom wavelength. Nano Lett. 9, 2935-2939 (2009).

43. Berini, P. \& Leon, I. D. Surface plasmon-polariton amplifiers and lasers. Nat. Phot. 6, 16-24 (2012).

44. Kéna-Cohen, S., Stavrinou, P., Bradley, D. \& Maier, S. Confined surface plasmonpolariton amplifiers. Nano Lett. 13, 1323-1329 (2013).

45. Kuppens, S. J. M., van Exter, M. P. \& Woerdman, J. P. Quantum-limited linewidth of a bad-cavity laser. Phys. Rev. Lett. 72, 3815-3818 (1994).

46. Colas des Francs, G. et al. Optical gain, spontaneous and stimulated emission of surface plasmon-polaritons in confined plasmonic waveguide. Opt. Exp. 18, 16327-16334 (2010)

47. Kim, K. Y., Tae, H.-S. \& Lee, J.-H. Analysis of leaky modes in circular dielectric rod waveguides. Electron. Lett. 39, 61-62 (2003).

48. Colas des Francs, G., Hugonin, J.-P. \& Čtyroký, J. Mode solvers for thin longrange plasmonics waveguides. Opt. Quant. Electron. 42, 557-570 (2011).

49. Snyder, A. \& Love, J. Optical Waveguide Theory (Chapman \& Hall, New York, 1983).

\section{Acknowledgements}

This work is supported by the Agence Nationale de la Recherche (under grants Plastips ANR-09-BLAN-0049, and HYNNA ANR-10-BLAN-1016), the French program Investissement d'Avenir (LABEX ACTION) and the Région de Bourgogne under the PARI-SMT3 program. Calculations were performed using DSI-CCUB resources (Université de Bourgogne).

\section{Author contributions}

G.C.F. supervised the project; G.C.F., J.B. and A.D. developed the formalism; J.B. performed the simulations and calculations; G.C.F., J.B. and A.B. discussed the results and wrote the manuscript.

\section{Additional information}

Supplementary information accompanies this paper at http://www.nature.com/ scientificreports

Competing financial interests: The authors declare no competing financial interests

How to cite this article: Barthes, J., Bouhelier, A., Dereux, A. \& Colas des Francs, G. Coupling of a dipolar emitter into one-dimensional surface plasmon. Sci. Rep. 3, 2734 DOI:10.1038/srep02734 (2013).

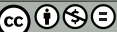

This work is licensed under a Creative Commons AttributionNonCommercial-NoDerivs 3.0 Unported license. To view a copy of this license, visit http://creativecommons.org/licenses/by-nc-nd/3.0 